\title{
AP4 is required for mitogen- and C-MYC-induced cell cycle progression
}

\author{
Rene Jackstadt ${ }^{1}$ and Heiko Hermeking ${ }^{1,2,3}$ \\ ${ }^{1}$ Experimental and Molecular Pathology, Institute of Pathology, Ludwig-Maximilians-Universität München, Munich, D-80337, \\ Germany \\ ${ }^{2}$ German Cancer Consortium (DKTK), Heidelberg, D-69120, Germany \\ ${ }^{3}$ German Cancer Research Center (DKFZ), Heidelberg, D-69120, Germany \\ Correspondence to: \\ Heiko Hermeking, e-mail: heiko.hermeking@med.uni-muenchen.de
}

Keywords: AP4, AP-4, TFAP4, C-MYC, cell cycle, cytokinesis

Received: August 8, $2014 \quad$ Revised and Accepted: August 11, $2014 \quad$ Published: October 8, 2014

\section{ABSTRACT}

\begin{abstract}
AP4 represents a C-MYC-inducible bHLH-LZ transcription factor, which displays elevated expression in many types of tumors. We found that serum-starved AP4deficient mouse embryo fibroblasts (MEFs) were unable to resume proliferation and showed a delayed S-phase entry after restimulation. Furthermore, they accumulated as tetraploid cells due to a cytokinesis defect. In addition, AP4 was required for c-MYC-induced cell cycle re-entry. AP4-deficient MEFs displayed decreased expression of CDK2 (cyclin-dependent kinase 2), which we characterized as a conserved and direct AP4 target. Activation of an AP4 estrogen receptor fusion protein (AP4-ER) enhanced proliferation of human diploid fibroblasts in a CDK2dependent manner. However, in contrast to C-MYC-ER, AP4-ER activation was not sufficient to induce cell cycle re-entry or apoptosis in serum-starved MEFs. AP4deficiency was accompanied by increased spontaneous and c-MYC-induced DNA damage in MEFs. Furthermore, C-MYC-induced apoptosis was decreased in AP4deficient MEFs, suggesting that induction of apoptosis by C-MYC is linked to its ability to activate AP4 and thereby cell cycle progression. Taken together, these results indicate that AP4 is a central mediator and coordinator of cell cycle progression in response to mitogenic signals and c-MYC activation. Therefore, inhibition of AP4 function may represent a therapeutic approach to block tumor cell proliferation.
\end{abstract}

\section{INTRODUCTION}

The AP4 protein belongs to the group of basichelix-loop-helix leucine zipper (bHLH-LZ) transcription factors [1]. AP4 exclusively forms homodimers, which bind to the E-box motif $\mathrm{CAG} / \mathrm{CCTG}$ and either repress or activate the expression of target genes [2-7]. We previously identified the AP4 gene as a direct transcriptional target of c-MYC and showed that the gene encoding the CDK-inhibitor $p 21$ is directly repressed by AP4 in human cells $[8,9]$. Recently, we also characterized the regulation of $p 21$ by AP4 in mouse embryonic fibroblasts (MEFs) and showed that AP4 also controls the expression of the CDK-inhibitor p16 in human and mouse cells [10]. In addition, we could demonstrate that deletion of AP4 leads to premature senescence and that $\mathrm{AP} 4$ is required for cellular transformation by c-MYC and mutant RAS [10]. Moreover, we performed a genome-wide analysis of AP4regulated genes and AP4 DNA binding in a human colon cancer (CRC) cell line [7]. Thereby we found that AP4 represents an epithelial-mesenchymal transition (EMT) inducing transcription factor (EMT-TF). We could further demonstrate that AP4 is crucial for metastases formation in a xenograft mouse model. Additionally, AP4 protein expression positively correlated with survival and distant metastases formation in the liver in two different colorectal cancer patient cohorts [7]. Moreover, elevated expression of AP4 correlated with poor patient survival also in gastric and hepatocellular cancer [11, 12]. Furthermore, we could identify a double-negative feedback-loop between AP4 
and the tumor-suppressive microRNAs miR-15a/16-1, which controls the balance of EMT and mesenchymal-epithelial transition (MET) during metastasis [13]. Recently, it has been shown that AP4 is a target for proteasomedependent degradation by the $\mathrm{SCF} / \beta \mathrm{TrCP}$ ubiquitin ligase [14]. This degradation was shown to be mediated by phosphorylation of AP4 on a conserved degron. Interestingly, the ectopic expression of a stable AP4 mutant revealed that $\beta \mathrm{TrCP}$-dependent degradation of AP4 is required for the fidelity of mitotic division.

The proto-oncogene $c-M Y C$ encodes a transcription factor of the bHLH-LZ class B, which binds to the E-box motif CACGTG (reviewed in [15]). c-MYC is commonly activated in human tumors via gene amplification, viral promoter insertion or chromosomal translocation, but also due to mutations of upstream regulators, such as APC/ adenomatosis polyposis coli and $\beta$-catenin (reviewed in [16]). $c-M Y C$ is highly expressed in proliferating cells and becomes down-regulated when cells cease to proliferate. Deregulated $c-M Y C$ expression promotes cell proliferation and causes resistance to anti-mitogenic stimuli [17]. Constitutive $c-M Y C$ expression sensitizes cells towards apoptosis (reviewed in [18]).

The mechanisms which underlie the mitogenicity of $c-M Y C$ are only partially understood. It seems likely that the combined actions of multiple genes regulated by $c-M Y C$ contribute to the stimulatory effects of $c-M Y C$ on cell cycle re-entry and progression [19]. Several c-MYCregulated genes encode components of the cell cycle machinery which control $\mathrm{G}_{1} / \mathrm{S}$-progression, such as cyclin D1/D2 [20, 21], CDK4 [22] and CDC25A [23] or represent regulators of the $\mathrm{G}_{2} / \mathrm{M}$ progression, as e.g. MAD2 [24]. Accordingly, c-MYC also influences $\mathrm{G}_{2} / \mathrm{M}$ progression $[24,25]$.

In order to determine the function of AP4 during cell cycle progression, we analyzed AP4-deficient MEFs. Our results imply that AP4 is, at least in MEFs, a required mediator of cell cycle progression after mitogenic stimulation and c-MYC activation. This function of AP4 is at least in part mediated by direct induction of the central cell cycle regulator CDK2. Our analyses further revealed that AP4 function is required for successful completion of the final steps of cell divisions, as AP4-deficient cells display a cytokinesis defect resulting in tetraploid cells.

\section{RESULTS}

\section{Role of AP4 in the mitogenic response}

In order to determine the function of AP4 during cell cycle progression we analyzed MEFs derived from AP4 knock-out mice, which we had generated by deletion of $A P 4$ exons 2-4 [10]. The resulting mice with $A P 4+/-$ and $A P 4-/-$ germ-line deletion did not display any overt phenotype and gave rise to offspring with normal Mendelian ratios (Hermeking et al., manuscript in preparation).
To generate MEFs, fibroblasts of embryos at day E13.5 were isolated and cultured as described previously [10]. In order to determine whether AP4 is involved in the proliferative response to mitogenic stimulation MEFs (passage 3) were kept at $0.5 \%$ serum for 24 hours and then re-stimulated by addition of medium with $10 \%$ serum (Figure $1 \mathrm{~A}$ ). AP4-deficient MEFs were unable to resume exponential proliferation in the following four days, whereas AP4+/+ MEFs immediately resumed exponential proliferation as determined by real-time impedance measurement. AP4+/MEFs showed an intermediate proliferative response. These results were confirmed by crystal-violet staining of identically treated MEFs 96 hours after re-stimulation (Figure 1B). Re-stimulated AP4-deficient cells displayed a pronounced delay of S-phase entry as determined by BrdUincorporation (Figure $1 \mathrm{C}$ and 1D). AP4+/- MEFs did not show a significant difference in the rate of BrdU-incorporation compared to $A P 4+/+$ MEFs. As the DNA-synthesis was delayed but not completely abolished in AP4-/- MEFs the proliferation defect described above presumably is not due to an inability to enter S-phase. As described below $A P 4+/+$ and $A P 4+/-$ MEFs display additional defects in cell cycle progression after mitogenic re-stimulation and an increase in double-strand DNA breaks, which may explain the inability to resume exponential proliferation. Taken together, AP4-deficient MEFs displayed a pronounced defect in their proliferative response after restimulation with serum which is presumably due to the deregulation of important cell cycle regulators in the absence of AP4.

\section{Characterization of $C D K 2$ as a direct AP4 target}

To identify factors involved in the proliferation defect caused by AP4 loss, we analyzed the expression of $\mathrm{CDK} 2$ as we recently identified $C D K 2$ as an AP4-target gene in a genome-wide screen for AP4-regulated genes in a human CRC cell line [7]. After re-stimulation, the induction of CDK2 was delayed and less pronounced in AP4-/MEFs, whereas the induction of CDK4 was not affected by AP4 loss (Figure 2A). As reported previously, the induction of CDK4 by re-stimulation with serum is mediated by c-MYC [22]. Therefore, the observed differential regulation of AP4 targets is presumably not indirectly caused by the delayed cell cycle re-entry of AP4-/- MEFs. As expected, expression of p21 protein was increased in AP4-deficient MEFs. Furthermore, in asynchronous cycling MEFs the CDK2 protein level was decreased in $A P 4$-deficient cells and showed intermediate expression in $A P 4+/-$ MEFs (Figure 2B). In addition, activation of a conditional AP4-ER (estrogen-receptor) fusion protein by addition of 4-OHT (4-hydroxy-tamoxifen) resulted in a rapid induction of CDK2 on the protein level in both, $A P 4+/+$ and AP4-/- MEFs (Figure 2C). By chromatin immunoprecipitation (ChIP) and subsequent qPCR analyses we could detect AP4 occupancy at an E-box present 350 bp up-stream of the CDK2 transcriptional start site 

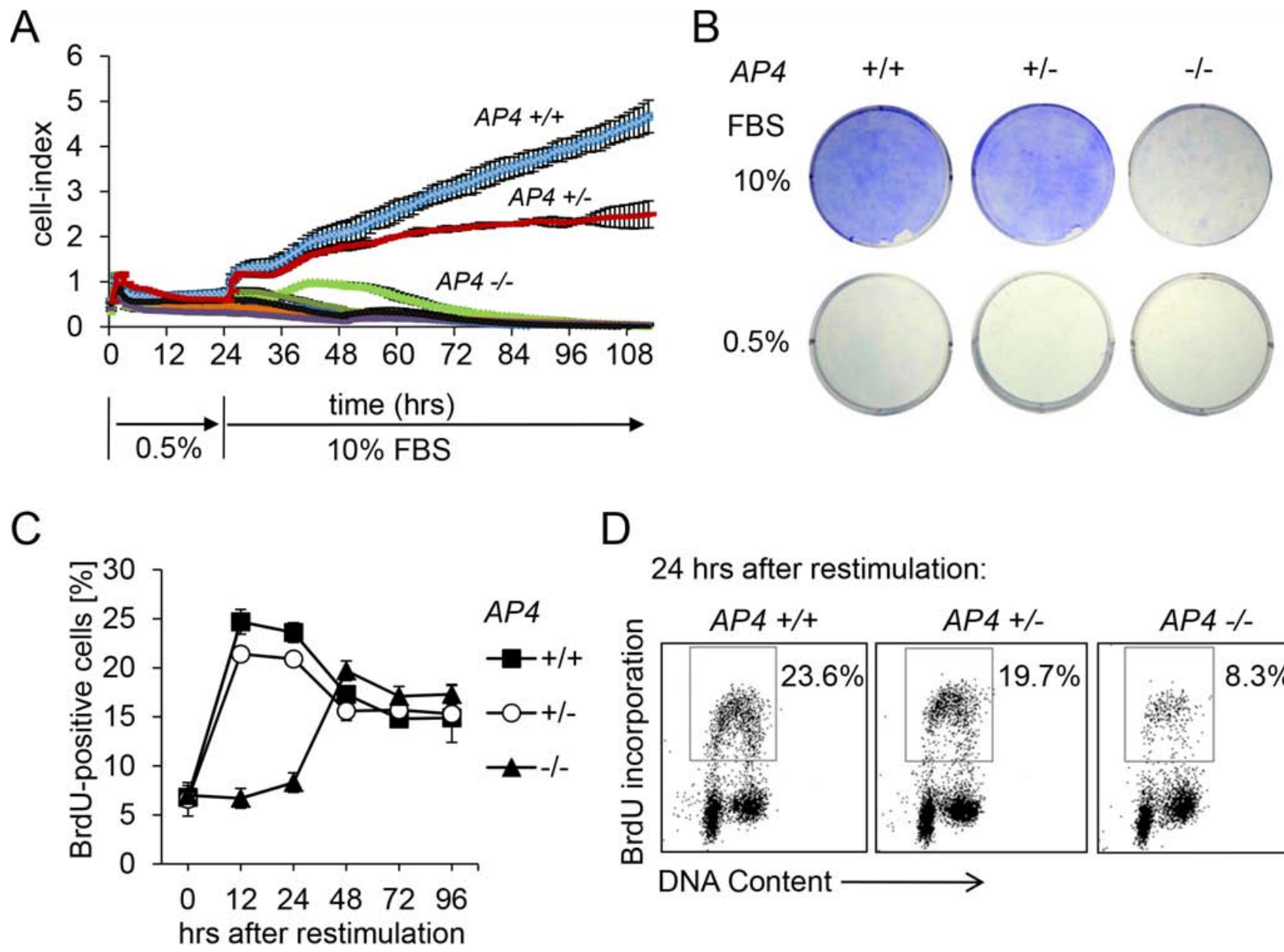

$24 \mathrm{hrs}$ after restimulation:

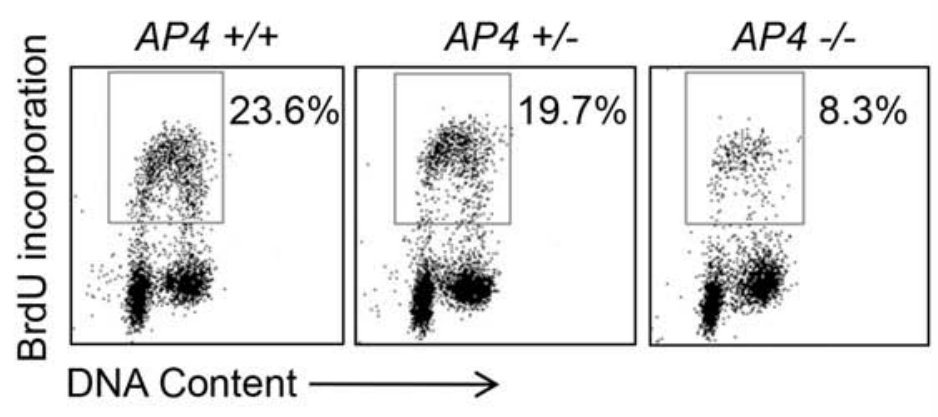

Figure 1: AP4 is required for proliferation after restimulation with serum. (A) Cell proliferation analysis by impedance measurement. MEFs (passage 3) with the indicated genotypes were cultivated for 24 hours at $0.5 \%$ serum before release into medium containing $10 \%$ serum. Control cells were continuously kept in $0.5 \%$ serum. $2 \times 10^{3}$ cells were seeded per well (96 well format) and analyzed over a period of 112 hours in one hour intervals. (B) $5 \times 10^{4}$ MEFs (passage 3) with the indicated genotype were seeded per well into a six well plate and cultivated for 24 hours at $0.5 \%$ serum before release into medium containing $10 \%$ serum for 96 hours. Subsequently cells were fixed and stained with crystal violet. (C) Determination of DNA-synthesis after re-stimulation as described in A and B. BrdU was added for the last hour. At the indicated time points cells were subjected to flow-cytometric analysis. (D) Exemplary flow-cytometric results of the quantification depicted in (C) 24 hours after restimulation. Results in A and C represent the mean $+/-\mathrm{SD}$ ( $\mathrm{n}=3$ ).

(TSS) in MEFs (Figure 2D and 2E). Two other E-boxes located $\sim 2.5 \mathrm{kbp}$ upstream did not display AP4 occupancy. Therefore, the mouse CDK2 gene represents a direct AP4 target gene.

\section{CDK2 is mediator of AP4-induced proliferation in HDFs}

The activation of an AP4-ER fusion protein by addition of 4-OHT also resulted in increased CDK2 protein levels in human diploid fibroblasts (HDFs) in the presence of $1 \%$ serum (Figure $3 \mathrm{~A}$ ). This was accompanied by hyper-phosphorylation of pRB (Figure 3B). Since hyper-phosphorylated RB is an indicator for a cell cycle re-entry we analyzed the BrdU incorporation as a measure of DNA replication after AP4 activation. Indeed, AP4 acti- vation at $1 \%$ serum led to enhanced DNA synthesis and proliferation, whereas AP4 activation was not sufficient to enhance proliferation at $0.25 \%$ serum (Figure 3C-E). Furthermore, treatment with the CDK2-inhibitor CVT313 [26] prevented the AP4-induced increase in proliferation at $1 \%$ serum (Figure $3 \mathrm{~F}$ ). These results show that CDK2 activation is required for AP4-mediated enhancement of proliferation in HDFs.

\section{AP4-deficiency results in a cytokinesis defect}

The analysis of the cell cycle distribution of re-stimulated AP4-deficient MEFs revealed a substantial shift towards the tetraploid (4N) state indicative of a $\mathrm{G}_{2} / \mathrm{M}$ arrest and a concomitant decrease of cells in the $\mathrm{G}_{1}$-phase (Figure 4A). We also observed an increase in apoptotic 
A

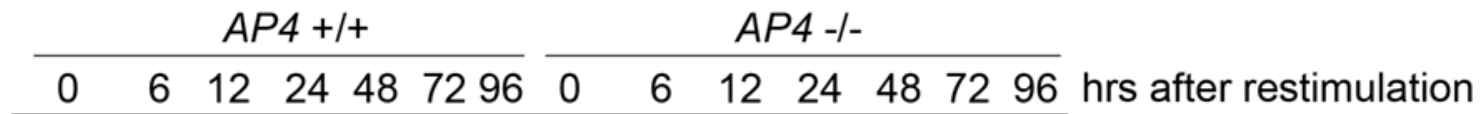

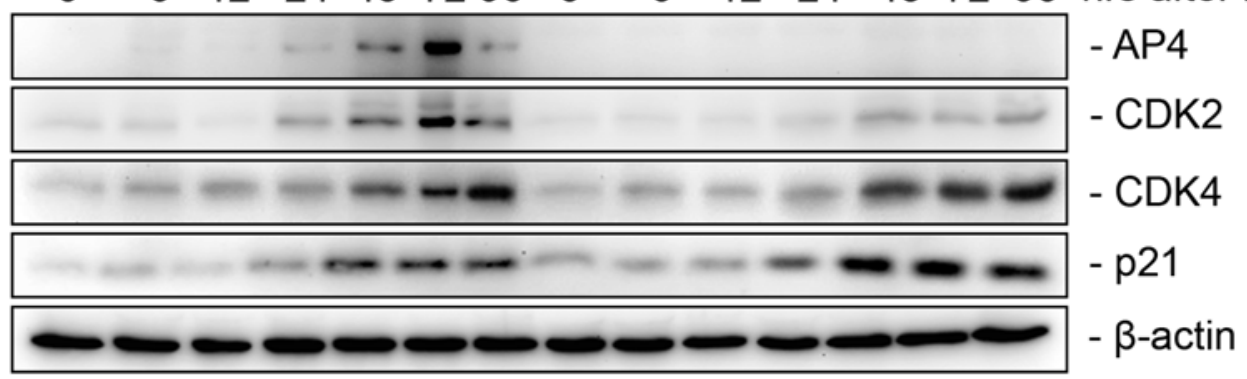

B
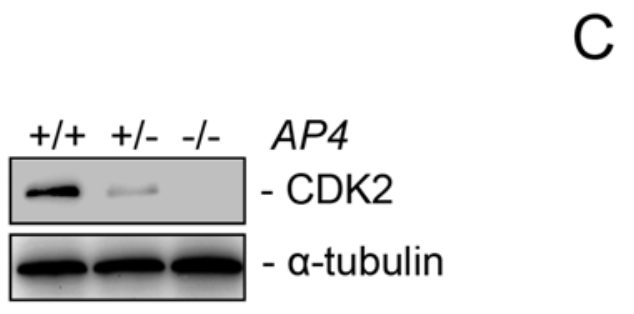

MEF/AP4-ER

$\frac{A P 4+/+}{0} 6 \frac{A P 4-/-}{1224} \frac{61224}{0}$ hrs $4-\mathrm{OHT}$

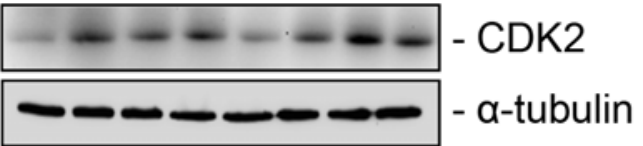

D
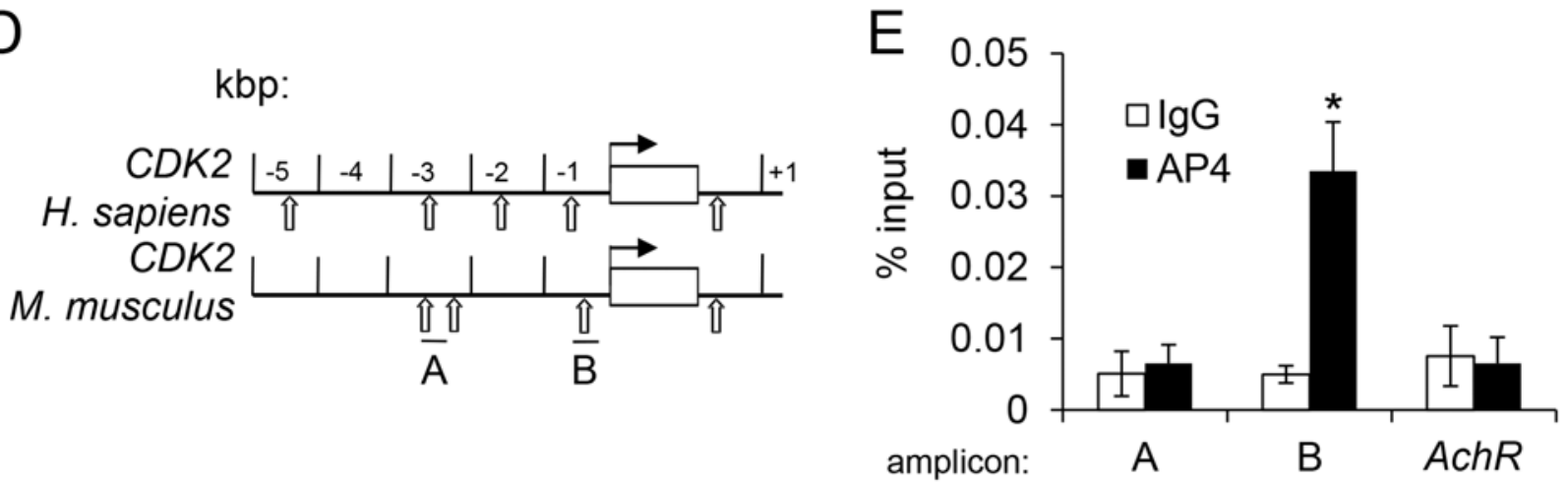

Figure 2: Characterization of murine $C D K 2$ as a direct target of AP4. (A) Immunoblot analysis of the respective proteins in MEFs (passage 3) with the indicated genotype harvested at the respective time-points after re-stimulation with $10 \%$ serum. $\beta$-actin served as loading control. (B) The expression of CDK2 was determined by immunoblot analysis in asynchronously growing MEFs (passage 3) of the indicated genotypes. $\alpha$-tubulin served as loading control. (C) Immunoblot analysis of the indicated proteins in MEFs with indicated genotypes after AP4-ER activation by addition of $200 \mathrm{nM}$ 4-OHT (4-hydroxy-tamoxifen) for the indicated periods. (D) Schematic representation of the genomic organization of the indicated human and murine promoters. Vertical arrows indicate AP4 binding motifs (CAGCTG). Horizontal bars indicate qChIP amplicons. (E) qChIP analysis of genomic DNA co-precipitated with an AP4-specific or mouse IgG control antibody in AP4+/+ MEFs. The mouse acetylcholine receptor (AchR) promoter lacking AP4 binding motifs served as a negative control. Results in D and F represent the mean $+/-\mathrm{SD}(\mathrm{n}=3)$. Significance level as indicated: ${ }^{*} \mathrm{p}<0.05$.

sub- $\mathrm{G}_{1}$ cells between 24 and 72 hours after restimulation of AP4-deficient MEFs. On the contrary, the majority of $A P 4+/+$ MEFs were in the $\mathrm{G}_{1}$-phase 96 hours after re-stimulation. Microscopic examination of the DAPIstained cells revealed that $A P 4-/-$ nuclei were generally enlarged and often showed invaginations and a butterflylike shape (Figure 4B). The latter is indicative for a failed cytokinesis. These formations were seen at intermediate frequencies in $A P 4+/-$ nuclei, but not in $A P 4+/+$ MEFs. Simultaneous detection of $\alpha$-tubulin and nuclear DNA further confirmed that AP4-deficient MEFs often display two connected nuclei in one cell body (Figure 4C). By using time-lapse video-microscopy we could record incomplete cytokinetic events resulting in enlarged, binucleated cells in AP4-/- MEFs but not in AP4+/+ MEFs (see exemplary pictures in Figure 4D and Movie S1). Presumably, the cells 
A

HDF/AP4-ER

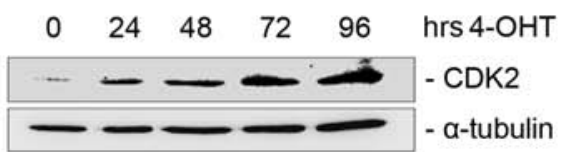

B

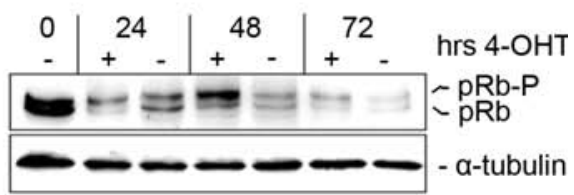

C
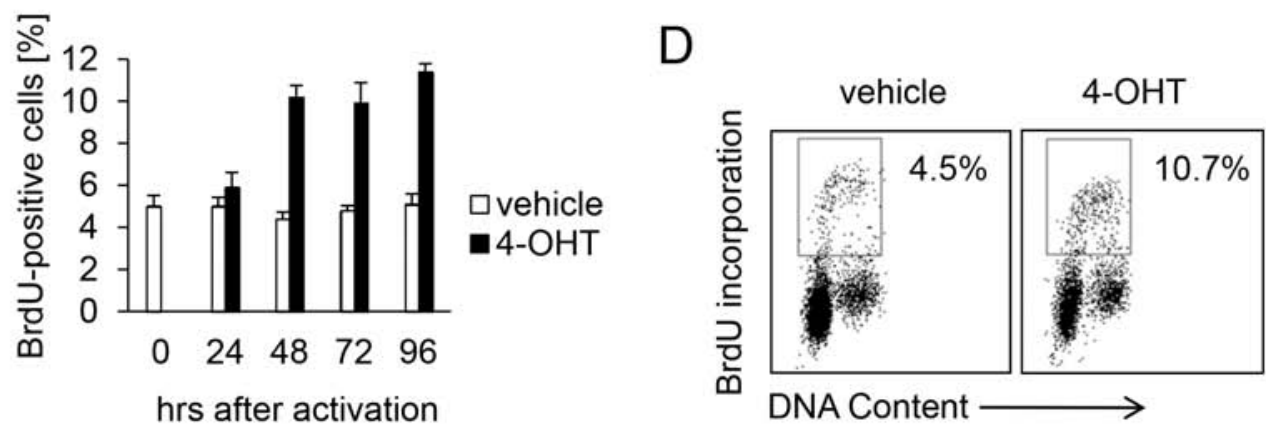

E
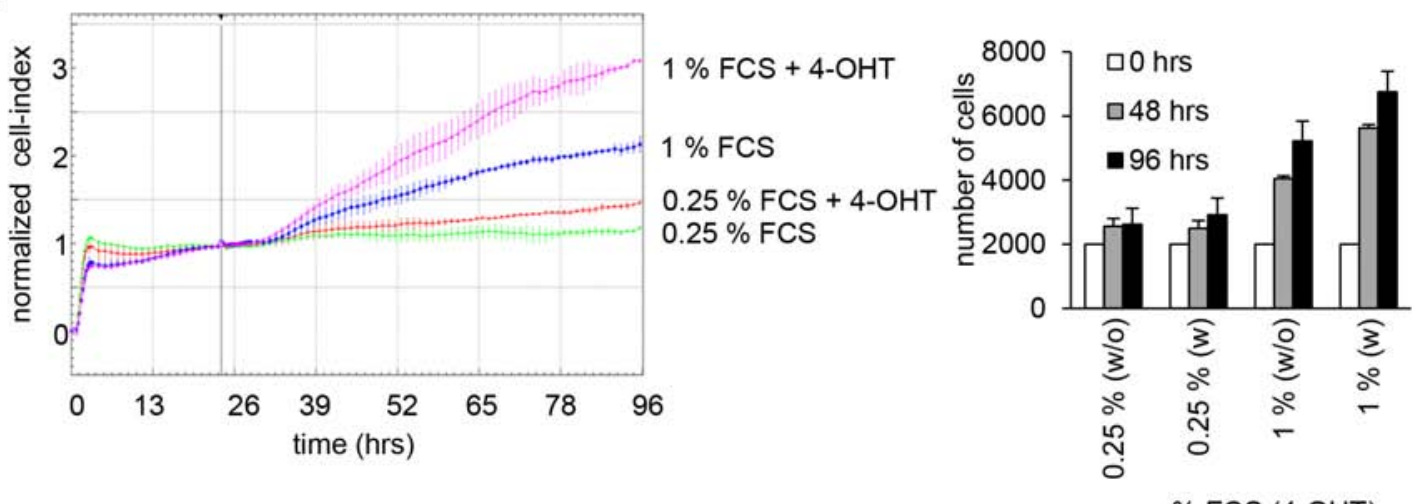

$\mathrm{F}$
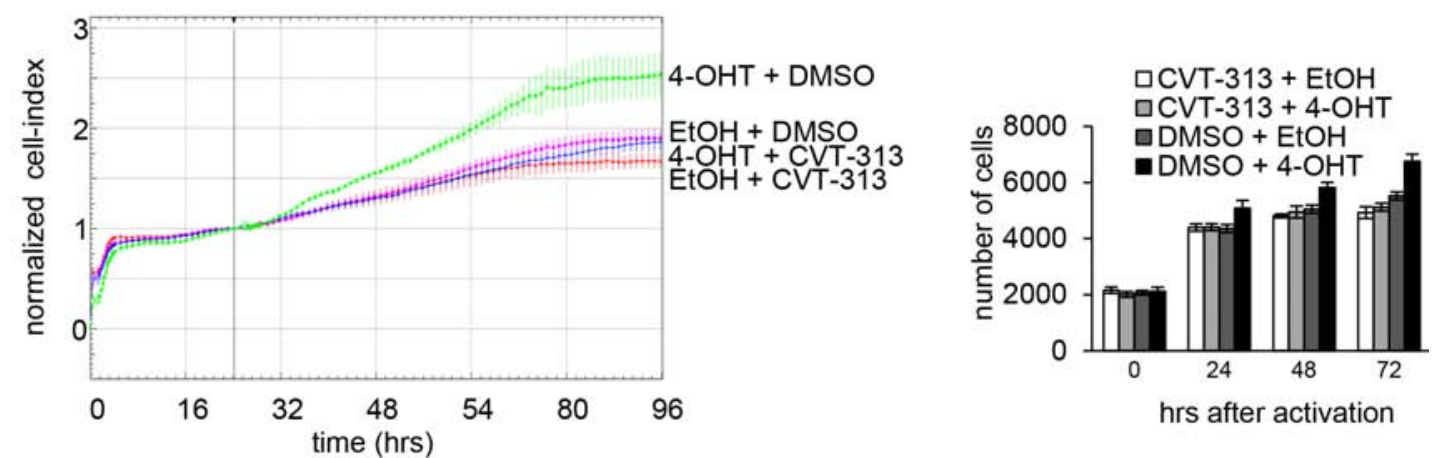

Figure 3: CDK2 is mediator of AP4-induced proliferation in HDFs. An AP4-ER fusion protein consisting of AP4 and the estrogen receptor (ER) was stably introduced into human diploid fibroblasts by retroviral transduction. HDFs were kept in $1 \%$ serum for 24 hours. Then AP4-ER was activated by addition of 4-OHT. (A) CDK2 and $\alpha$-tubulin protein expression was detected by immunoblotting after activation of AP4-ER for the indicated periods. (B) Detection of active, hypo-phosphorylated pRB and inactive, hyper-phosphorylated pRb-p protein by immunoblotting after activation of AP4-ER for the indicated periods. (C) Analysis of de novo DNA-synthesis after activation of AP4-ER by flow-cytometry. HDFs were kept in 1\% serum for 24 hours before activation of AP4-ER by addition of 4-OHT (200 nM) for the indicated periods. Results represent the mean $+/-\mathrm{SE}(\mathrm{n}=2)$. (D) Exemplary flow-cytometric results of the quantification depicted in (C) 96 hours after AP4-ER activation. (E) The respective cells were cultivated in the presence of the indicated serum concentrations for 24 hours before addition of 4-OHT. Cell proliferation was determined by impedance measurement (left panel) or by standard cell counting with Trypan blue exclusion (right panel). Results represent the mean $+/-\mathrm{SD}(\mathrm{n}=3)$. (F) Effect of CDK2 inhibition on AP4-induced proliferation. The cells were kept in $1 \%$ serum for 24 hours and then treated with the indicated combinations of the specific CDK2-inhibitor CVT-313 (0.5 $\mu \mathrm{M})$, inhibitor-vehicle (DMSO), 4-OHT (200 nM) and its vehicle (EtOH). Experiments in (C, E and F) were carried out in triplicates $(\mathrm{n}=3)$. 
A
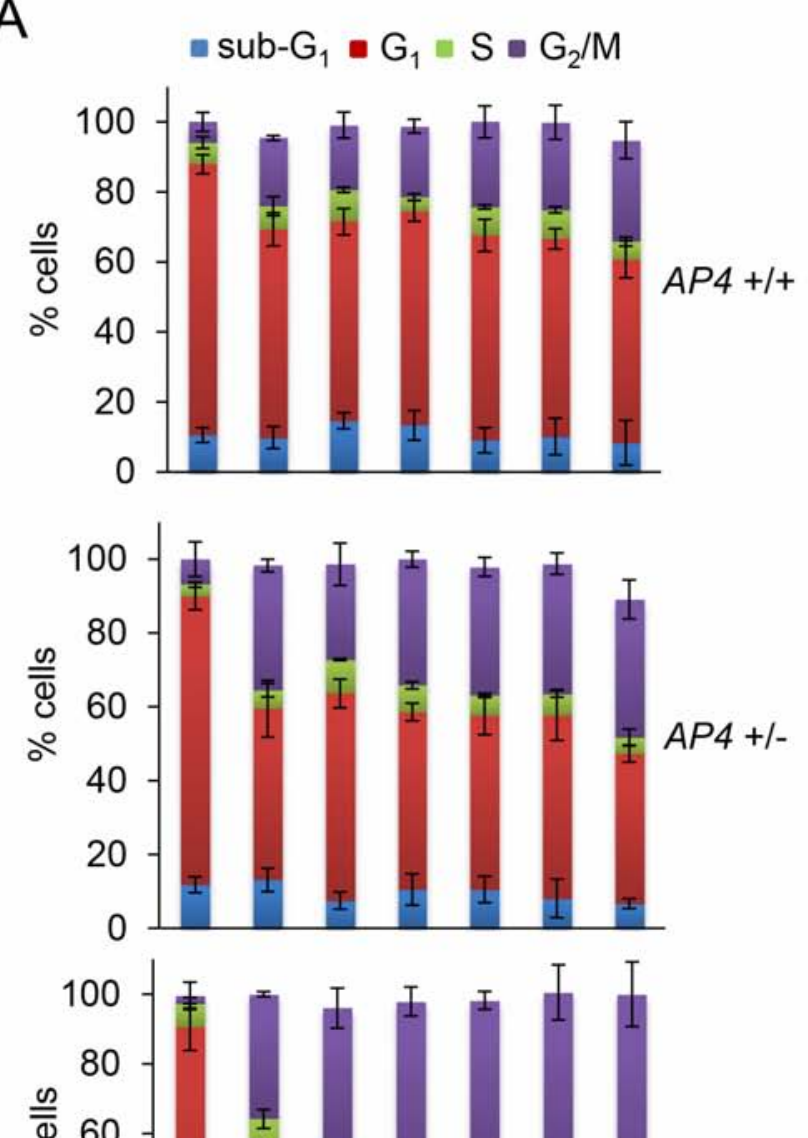

AP4 - / D

C

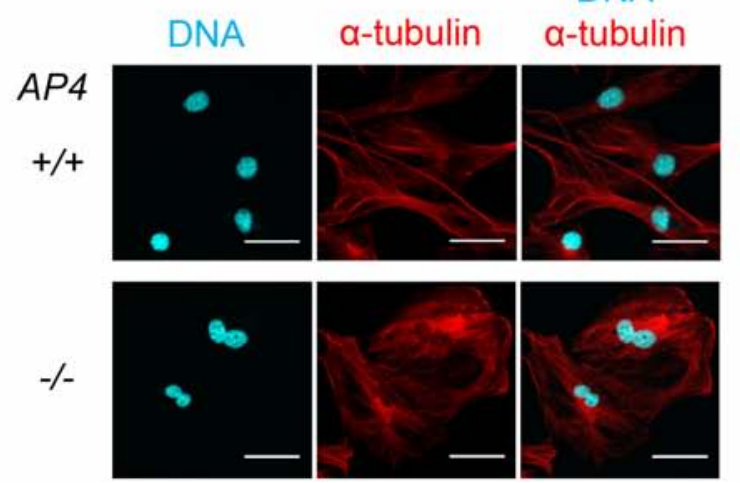

B

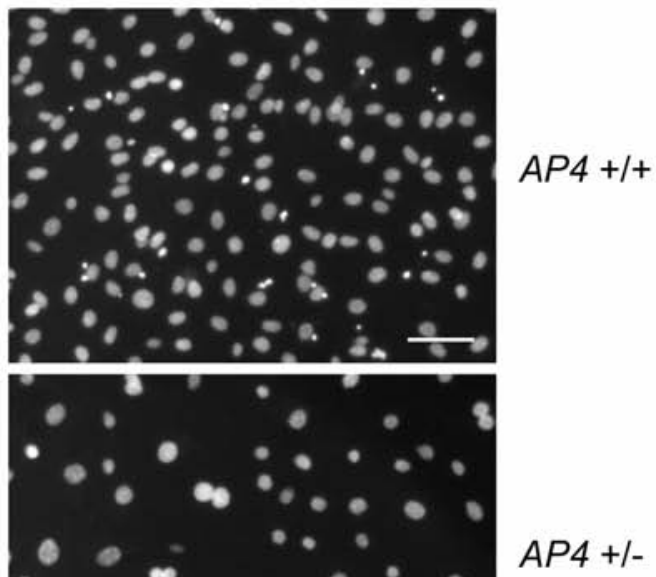

AP4 -/-
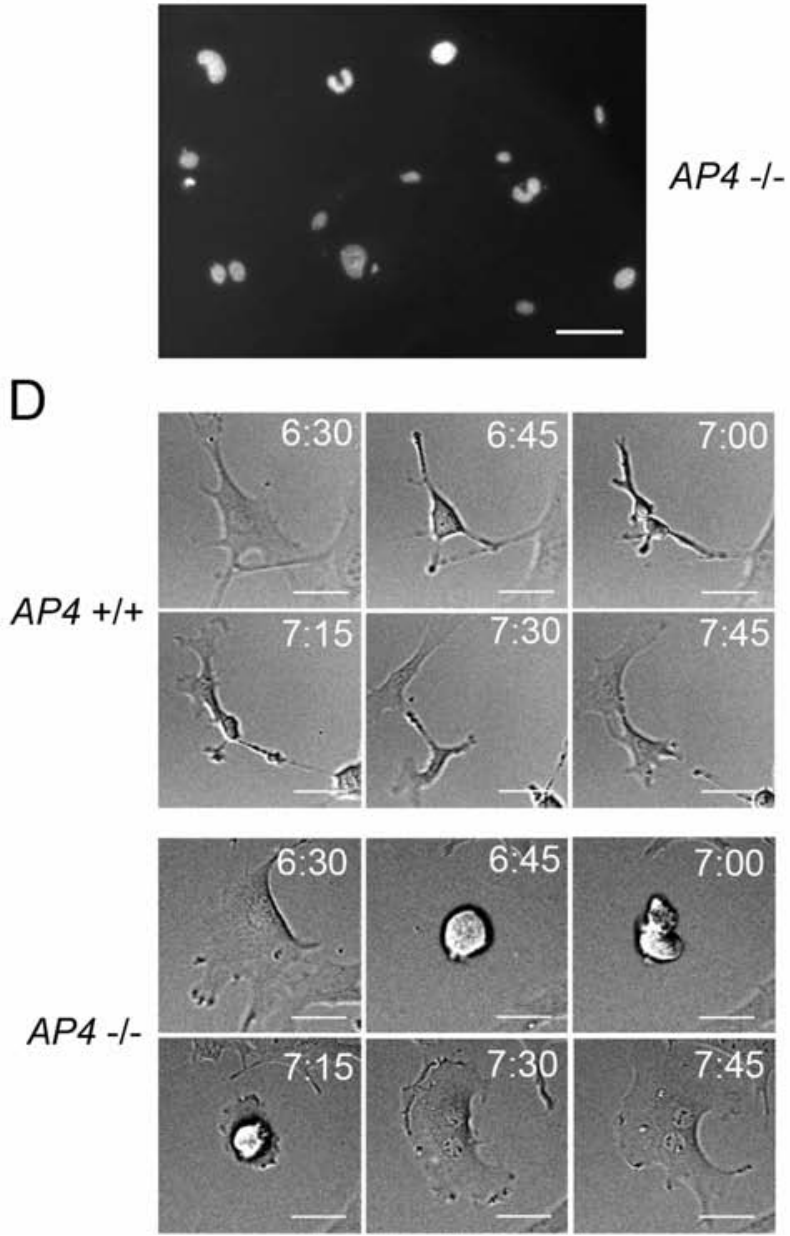

Figure 4: AP4-deficiency results in a cytokinesis defect. (A) Determination of cell cycle distribution by DNA content analysis after release of serum-starved ( $0.5 \%$ FBS for 24 hours) MEFs (passage 3) into $10 \%$ serum containing medium. Per $25 \mathrm{~cm}^{2}$ flask $5 \times 10^{4}$ MEFs with the indicated genotypes were analyzed. Results represent the mean $+/-$ SD ( $=3$ ). (B) Nuclear DNA of MEFs (passage 3) treated as in (A) 96 hours after re-stimulationwas stained with DAPI and microscopy pictures were taken. The scale bar represents $10 \mu \mathrm{m}$. (C) Microscopic analysis of starved (0.5\% FBS for 24 hours) MEFs (passage 3) with the indicated genotypes 96 hours after re-stimulation with $10 \%$ serum. Nuclear DNA was visualized by DAPI (cyan) and $\alpha$-tubulin staining by indirect immunofluorescence (red). The scale bar represents $10 \mu \mathrm{m}$. (D) Representative picture sequences of MEFs recorded by time-lapse video-microscopy after re-stimulation with $10 \%$ serum. The time elapsed after re-stimulation is indicated in hours. The scale bar represents $10 \mu \mathrm{m}$. 
resulting from such events are arrested in a $\mathrm{G}_{1}$-like state with a $4 \mathrm{~N}$ DNA content. These results show that AP4 not only regulates the $\mathrm{G}_{1} / \mathrm{S}$ transition, but also has functions during and/or affects the $\mathrm{G}_{2} / \mathrm{M}$ transition.

\section{AP4 as a mediator of c-MYC function}

We could previously demonstrate a direct regulation of AP4 by c-MYC $[8,10]$. Therefore, we determined the role of AP4 during c-MYC-induced cell proliferation and apoptosis in MEFs with $A P 4-/-$ and $A P 4+/+$ genotypes. c-MYC-ER activation in serum-starved MEFs resulted in a more than three-fold increase in BrdU incorporation and therefore DNA-replication in $\mathrm{AP} 4+/+$ MEFs, whereas AP4-/- MEFs showed no significant increase in BrdU incorporation 24 hours after c-MYC activation (Figure 5A). AP4-ER activation in $A P 4+/+$ or in $A P 4-/-$ MEFs did not results in a increase in DNA synthesis (Figure 5A). The relative increase in apoptosis after c-MYC-ER activation in serum starved MEFs was strongly decreased in AP4-deficient MEFs (Figure 5B): upon c-MYC activation apoptosis in $A P 4+/+$ MEFs increased from $\sim 12 \%$ to $\sim 24 \%$ and therefore doubled, whereas $A P 4-/-$ MEFs only displayed a minor increase in apoptosis.
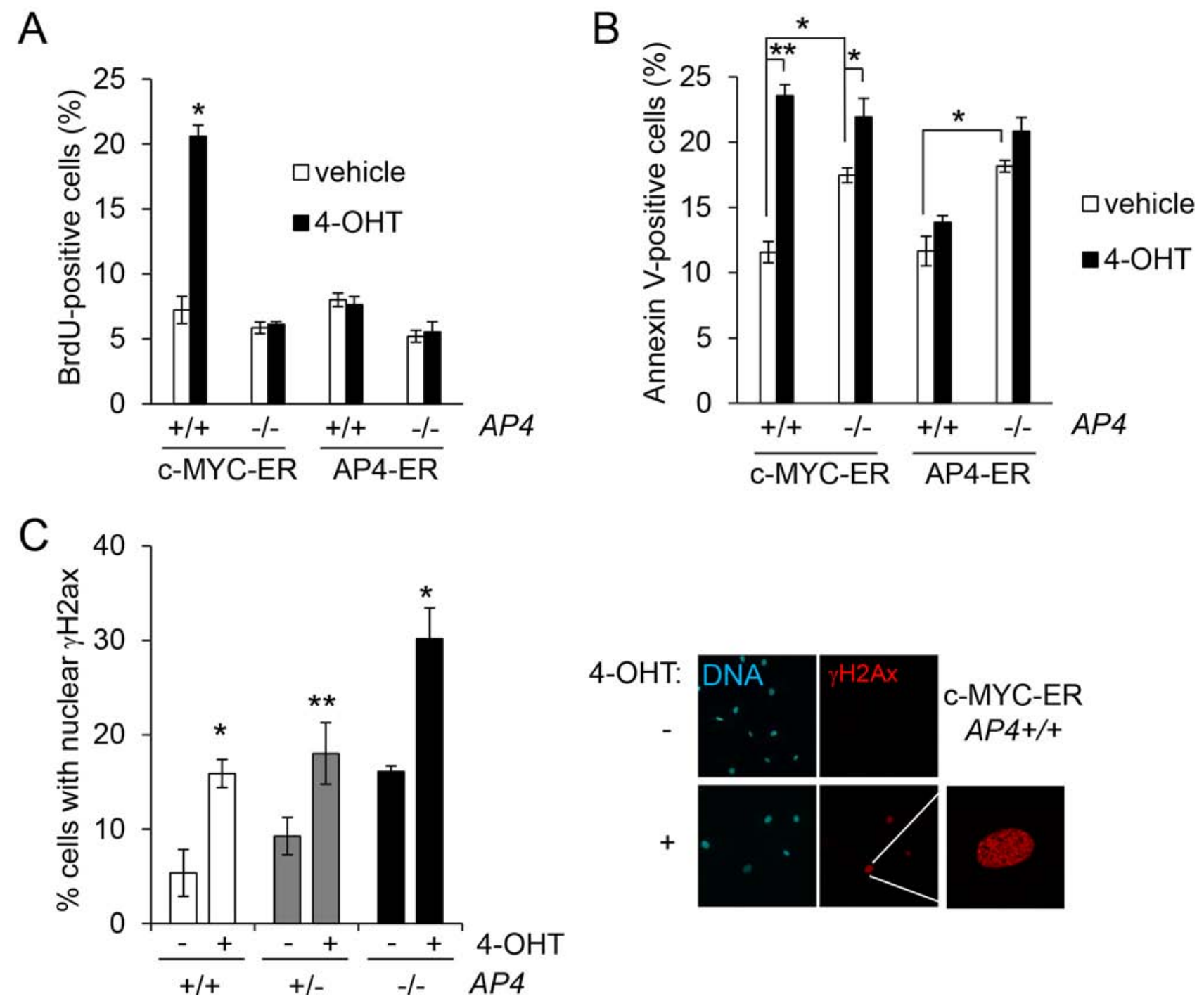

Figure 5: AP4 as a mediator of c-MYC function. (A) Quantification of de novo DNA-synthesis by BrdU incorporation after c-MYC-ER or AP4-ER activation by addition of $200 \mathrm{nM} 4-\mathrm{OHT}$ for 24 hours. MEFs (passage 5) of the indicated genotype stably expressing virally transduced c-MYC-ER or AP4-ER were kept for 48 hours at $0.5 \%$ serum before the addition of 4-OHT. (B) Quantification of apoptotic cells by Annexin V staining, 48 hours after c-MYC-ER or AP4-ER activation. (C) Quantification of $\gamma \mathrm{H} 2 \mathrm{Ax}$-positive nuclei by immunofluorescence 24 hours after c-MYC-ER activation in the indicated MEFs. $\gamma \mathrm{H} 2 \mathrm{Ax}$ was detected with a specific antibody and an Alexa-555-labeled secondary antibody, nuclei by DAPI staining. For every condition and genotype biological triplicates were analyzed microscopically with ten different fields for each replicate. Right panel, exemplary results of the described analyses. Results in (A-D) represent the mean $+/-\mathrm{SD}(\mathrm{n}=3)$. Significance level as indicated: ${ }^{*} \mathrm{p}<0.05, * * \mathrm{p}<0.01$ 
Therefore, AP4 is required for c-MYC-induced DNAsynthesis and, at least in part, for c-MYC-induced apoptosis. These results furthermore support the notion that c-MYC-induced apoptosis is linked to c-MYC-induced cell cycle progression. In addition, ectopic AP4 expression is not sufficient, at least in serum-starved MEFs, for the induction of cell cycle re-entry and apoptosis.

Furthermore, AP4-/- MEFs showed increased levels of spontaneous apoptosis after serum starvation (Figure 5B; bars representing cells treated with vehicle). This phenotype might be due to the increased level of spontaneous DNA damage that we observed in AP4-deficient MEFs, as evidenced by an increase of nuclear foci of $\gamma \mathrm{H} 2 \mathrm{Ax}$ (Figure 5C), which represent sites of doublestranded DNA breaks and/or replication fork collapses [27]: whereas $\sim 5 \%$ of serum-starved wild-type MEFs displayed nuclear $\gamma \mathrm{H} 2 \mathrm{Ax}$ signals, $\sim 17 \%$ of the AP4-deficient MEFs were positive for $\gamma \mathrm{H} 2 \mathrm{Ax}$. After c-MYC-ER activation a two-to-three fold increase of $\gamma \mathrm{H} 2 \mathrm{Ax}$-positive nuclei was observed irrespective of the AP4 genotype. However, the frequency of $\gamma \mathrm{H} 2 \mathrm{Ax}$-positive nuclei reached $\sim 30 \%$ in AP4-/- MEFs, whereas AP4+/+ MEFs showed $\sim 16 \%$ $\gamma \mathrm{H} 2 \mathrm{Ax}$-positive nuclei after c-MYC activation. Taken together, these results show that AP4 is necessary for an effective re-entry into the cell cycle after c-MYC activation. In the absence of $A P 4$, the lack of AP4-mediated gene regulations may results in an increase in replicationrelated DNA damage, which interferes with the c-MYCinduced progression from $\mathrm{G}_{1}$ - to S-phase. Therefore, AP4 is required for a coordinated cell cycle re-entry after c-MYC activation.

\section{DISCUSSION}

By analysis of AP4-deficient MEFs we were able to identify new and important cellular functions of the bHLH-LZ transcription factor AP4. We found that AP4 is necessary for c-MYC-induced cell cycle re-entry and mitogen-induced cell cycle progression in MEFs, presumably by controlling the expression of the central cell cycle regulator $\mathrm{CDK} 2$. Our analyses also revealed that AP4 function is required during the $\mathrm{G}_{2} / \mathrm{M}$ transition, as $A P 4$ deficient cells displayed a cytokinesis defect resulting in tetraploid cells.

Similar to the AP4-deficient MEFs analyzed here, $C D K 2-/-$ MEFs display a cell-cycle re-entry defect $[28,29]$. Interestingly, $C D K 4$-deficient MEFs also display a delayed entry into $\mathrm{S}$-phase after serum re-stimulation $[30,31]$. Presumably, AP4 regulates the activity of CDK4 by repression of the two CDK-inhibitors p16 and $\mathrm{p} 21$. Taken together, it is possible that the defects in S-phase reentry and the premature senescence observed in AP4-negative cells are due to the decreased expression of CDK2 combined with elevated p21 and p16 levels, which inhibit the activity of the remaining $\mathrm{CDK} 2 /$ cyclin and other CDK/ cyclin complexes.
The increase in spontaneous dsDNA breaks in AP4-deficient MEFs presumably results from replication stress caused by uncoordinated cell cycle events that may occur in the absence of AP4. The cytokinesis failure and subsequent accumulation of binucleated, tetraploid cells in AP4-deficient MEFs may be caused by aberrant events that occurred in the earlier phases of the cell cycle. For example, the elevated frequency of dsDNA breaks as evidenced by the increased number of $\gamma \mathrm{H} 2 \mathrm{Ax}$ foci in AP4-deficient cells can trigger pathways that regulate the expression of cytokinesis proteins, or their activity by post-translational modification and thereby block cytokinesis completion as discussed in [32]. The elevated expression of the two CDK inhibitors p16 and p21 in combination with the decreased expression of CDK2 might also contribute to the cytokinesis failure in AP4deficient cells. For example, altered CDK activity was shown to affect centrosome duplication, which may result in mono- or multi-polar spindles, which ultimately may cause a defective cytokinesis [33-36].

In the future it will be important to further evaluate the role of AP4 in cell proliferation in models of c-MYCinduced tumorigenesis. In addition, the aberrant molecular processes underlying in the cell cycle defects of AP4deficient cells that were described here deserve further analysis. Furthermore, our results suggest that inhibition of AP4 function, e.g. by small molecules blocking AP4 homodimerization, may be employed to therapeutically target c-MYC-driven tumor cells.

\section{EXPERIMENTAL PROCEDURES}

\section{Generation of $\boldsymbol{A P 4}$-deficient mice}

AP4-deficient mice and their phenotypes will be described elsewhere (Hermeking et al., in preparation). As described previously [10], the loxP-site flanked exons 2-4 of AP4 were removed by crossing the respective mice with CMV-Cre mice. All ES cells and mice used had a $\mathrm{C} 57 \mathrm{Bl} / 6$ background. The Cre allele was removed by further crossing. The respective genotypes of the mice and MEFs were confirmed by specific PCR analyses. Primers sequences for genotyping can be found in [10].

\section{Isolation and cultivation of mouse embryonic fibroblasts and human diploid fibroblasts}

MEFs were isolated from day E13.5 embryos of the mice described above. The uterus was removed and washed in PBS. The yolk sacs were separated and the embryos were isolated carefully. The viscera of each embryo was removed and the embryo was washed twice in PBS, placed in trypsin-EDTA (Invitrogen), cut into smaller pieces and incubated for 10 minutes at $37^{\circ} \mathrm{C}$. The cell suspension prepared from the embryo 
was washed with medium containing 10\% FBS and plated in $10 \mathrm{~cm}$ culture dishes. MEFs and HDFs were routinely cultured in a humidified $5 \% \mathrm{CO}_{2}$ and $20 \% \mathrm{O}_{2}$ atmosphere at $37^{\circ} \mathrm{C}$. MEFs and HDFs were maintained in Dulbecco's Modified Eagles Medium (DMEM, Invitrogen) containing $10 \%$ fetal bovine serum (FBS). MEFs were cultivated in presence of 100 units $/ \mathrm{ml}$ penicillin and $0.1 \mathrm{mg} / \mathrm{ml}$ streptomycin. One passage represents treatment with trypsin and subsequent dilution of the cells at a ratio of 1:3 every three days. 4-hydroxytamoxifen (4-OHT, Sigma) was dissolved in ethanol (400 $\mu \mathrm{M}$ stock solution) and used at a final concentration of $200 \mathrm{nM}$. MEFs used in the same analysis were derived from littermates.

\section{Western blot analysis and antibodies}

Cells were lysed in RIPA lysis buffer $(50 \mathrm{mM}$ Tris/ $\mathrm{HCl} \mathrm{pH} 8.0,250 \mathrm{mM} \mathrm{NaCl}, 1 \% \mathrm{NP} 40,0.5 \%$ (w/v) sodium deoxycholate, $0.1 \%$ sodium dodecylsulfate, complete mini protease inhibitors (Roche)). Lysates were sonicated and centrifuged at $16.060 \mathrm{x}$ g for 15 minutes at $4{ }^{\circ} \mathrm{C}$. Per lane $30-80 \mu \mathrm{g}$ of whole cell lysate was separated using $7.5 \%$ or $12 \%$ SDS-acrylamide gels and transferred on Immobilon PVDF membranes (Millipore). For immuno-detection membranes were incubated with antibodies specific for CDK2 (Santa Cruz), CDK4 (Santa Cruz), p21 (BD PharMingen), RB (BD PharMingen), $\beta$-actin (Sigma) or $\alpha$-tubulin (Sigma). Signals from HRP (horse-radish-peroxidase)-coupled secondary antibodies were generated by enhanced chemiluminesence (Perkin Elmer Life Sciences, Boston, MA) and recorded with a CCD camera (440CF imaging system, Eastman Kodak Co., Rochester, NY).

\section{Retroviral infections}

For retrovirus production and infection of MEFs Phoenix-E and for HDF cells Phoenix-A packaging cells were transfected with the respective $\mathrm{pBabe}$ vectors using calcium phosphate precipitation. Twenty-four hours after transfection, retrovirus-containing supernatants were harvested, passed through $0.45 \mu \mathrm{m}$ filters (Millipore) and used to infect MEFs or HDFs in the presence of polybrene $(8 \mu \mathrm{g} / \mathrm{ml})$ four times in four hour intervals. Selection was started 48 hours later by addition of $2 \mu \mathrm{g} / \mathrm{ml}$ puromycin (Sigma) for five days.

\section{Phase-contrast microscopy}

Images of cells in culture were captured using an Axiovert 25 microscope (Zeiss) with a Sony Digital Hyper HAD camera (Software: Kappa Image Base, Kappa Optoelectronics) or an Axiovert Observer Z.1 microscope connected to an AxioCam MRm camera with an Axiovision software (Zeiss).

\section{Plasmids}

The generation of the plasmids used here was previously described in [10]. The pBabe-MYC-ER construct was kindly provided by Bruno Amati (Milan).

\section{Flow cytometric analysis of DNA synthesis and DNA content}

To monitor DNA synthesis $50 \mu \mathrm{M}$ 5-bromo-2'deoxyuridine (BrdU, Roche) was added for 60 minutes at $37^{\circ} \mathrm{C}$. Next, cells were harvested by addition of trypsin, resuspended and centrifuged at $300 \mathrm{~g}$ for five minutes. After washing with phosphate buffered saline (PBS) cells were fixed by addition of ice-cold $70 \%$ ethanol and incubation for at least 30 minutes at $-20^{\circ} \mathrm{C}$. Fixed cells were resuspended in $0.1 \mathrm{mg} / \mathrm{ml}$ pepsin and DNA was denatured by incubation in $2 \mathrm{M} \mathrm{HCl}$ for $30 \mathrm{~min}$ at room temperature. After centrifugation $(500 \mathrm{~g})$ cells were resuspended in $0.1 \mathrm{M} \mathrm{Na}_{2} \mathrm{~B}_{4} \mathrm{O}_{7}$. Cells were washed once with PBS and PTS buffer (PBS, $0.5 \%$ Tween 20, 2\% FBS) respectively, and subsequently resuspended in $60 \mu \mathrm{l}$ PTS $+6 \mu \mathrm{l}$ antiBrdU-FITC antibody (BD Biosciences Pharmingen) or an appropriate isotype control $\mathrm{IgG}$ and incubated for $30 \mathrm{~min}$ at room temperature in the dark. Next, cells were washed two times with PTS and resuspended in $500 \mu \mathrm{PTS}, 0.5$ $\mathrm{mg} / \mathrm{ml} \mathrm{RNase} \mathrm{A} \mathrm{(Sigma)} \mathrm{and} 50 \mu \mathrm{g} / \mathrm{ml}$ propidium iodide (Sigma). After incubation for $30 \mathrm{~min}$ at RT cells were analyzed by flow cytometry (CFlow6, Accuri). For DNA content analysis cells were harvested by addition of trypsin, washed and stained with a propidium iodide solution as described in [13]. Afterwards, cells were subjected to flow cytometry using a CFlow6 device (Accuri).

\section{Chromatin immunoprecipitation (ChIP) assay}

Cross-linking was performed with formaldehyde (Merck) at a final concentration of $1 \%$ and terminated after five minutes by addition of glycine at a final concentration of $0.125 \mathrm{M}$. Cells were harvested with SDS buffer (50 mM Tris pH 8.1, 0.5\% SDS, $100 \mathrm{mM} \mathrm{NaCl}$, $5 \mathrm{mM}$ EDTA) and resuspended in IP buffer after pelleting (2 parts of SDS buffer and 1 part Triton dilution buffer (100 mM Tris- $\mathrm{HCl} \mathrm{pH} 8.6,100 \mathrm{mM} \mathrm{NaCl}, 5 \mathrm{mM}$ EDTA, $\mathrm{pH} 8.0,0.2 \% \mathrm{NaN}_{3}, 5.0 \%$ Triton $\left.\left.\mathrm{X}-100\right)\right)$. Chromatin was sheered by sonication (HTU SONI 130, G. Heinemann) to generate DNA fragments with an average size of $500 \mathrm{bp}$. Preclearing and the incubation with AP4 antibody (AbD Serotec) or the respective IgG control (M-7023, Sigma) for 16 hours was performed as previously described [24]. Washing and reversal of cross-linking was performed as described [37]. Immunoprecipitated DNA was analyzed by qPCR and the enrichment was expressed as percentage of the input for each condition [37]. The sequences of oligonucleotides used as qChIP primers are listed in Table S1. 


\section{Crystal violet staining}

Cells were washed with PBS and fixed with 70\% $\mathrm{EtOH}$ for 20 minutes at room temperature. Cells were stained with a crystal violet solution $(5 \mathrm{mg} / \mathrm{ml}$ crystal violet (Sigma)) in 20\% methanol for 30 minutes at room temperature. Then cells were washed extensively with water and the whole plate was photographed.

\section{Live cell imaging}

After 24 hours of serum starvation with $0.5 \%$ serum MEFs were restimulated with a final concentration of $10 \%$ serum. 6 hours after restimulation the recording was initiated and performed for 42 hours in intervals of 15 minutes. For this an Axiovert Observer Z.1 microscope with an AxioCam MRm camera controlled by the Axiovision software (Zeiss) was used. Movies were recorded with a Plan-Apochromat $20 \mathrm{x} / 0.8 \mathrm{M} 27$ objective (Zeiss) at $37^{\circ} \mathrm{C}$ and $5 \% \mathrm{CO}_{2}$.

\section{Assessment of proliferation by impedance measurements}

The optimal cell concentration was determined by serial dilution for each cell line. For MEFs and HDFs $2 \times 10^{3}$ cells were seeded per well (96-well). Subsequently, $100 \mu \mathrm{l}$ of cell culture media at room temperature was added into each well of an E-plate 16 (Roche). Proper electrical-contacts and background impedance was measured. $100 \mu \mathrm{l}$ cell suspension was added to medium containing wells on the E-plate 16. After 30 minutes incubation at room temperature the E-plate 16 was placed in a Xcelligence device (Roche) in a cell culture incubator. Impedance was monitored every 60 minutes for a period of up to 110 hours. The electrical impedance is represented as a dimension-less parameter termed cell-index (CI). All calculations were performed using the RTCA-integrated software of the Xcelligence system.

The unit-less parameter CI represents relative changes in electrical impedance caused by cells adhering to the surface of the wells which is calculated based on the following formula: $\mathrm{CI}=(Z \mathrm{i}-Z 0) / 15$, where $Z \mathrm{i}$ is the impedance at an individual point of time during the experiment and $Z 0$ is the impedance at the start of the experiment. Impedance is measured at 3 different frequencies $(10,25$ or $50 \mathrm{kHz})$ and a specific time (ref: Roche Diagnostics GmbH. Introduction of the RTCA DP Instrument. RTCA DP Instrument Operator's Manual, A. Acea Biosciences, Inc.; 2008.). To validate impedance measurements, cells were also seeded into 96 well plates in triplicates and counted at the indicated time points using a Neubauer-chamber.

\section{Apoptosis Detection with Annexin V}

The protocol was performed according to the manufacturer's instructions (BD Pharmingen, 556570). Briefly, $8 \times 10^{4}$ cells harboring c-MYC-ER or AP4-ER were seeded into a $25 \mathrm{~cm}^{2}$ flask. Cells were serum starved for 48 hours with $0.5 \%$ serum. For activation of c-MYC or AP4 4-OHT $(200 \mathrm{nM})$ or the vehicle (ethanol) was added. After 48 hours cells were harvested by trypsination and washed twice with PBS. Cells were resuspended in $1 \mathrm{x}$ bindingbuffer (0.01 M Hepes/NaOH (pH 7.4), 0.14 M NaCl, 2.5 $\mathrm{mM} \mathrm{CaCl}{ }_{2}$ ) at a concentration of $1 \times 10^{6}$ cells $/ \mathrm{ml} .100 \mu \mathrm{l}$ of the solution $\left(1 \times 10^{5}\right.$ cells $)$ were incubated with $5 \mu \mathrm{l}$ of FITC Annexin V and $5 \mu \mathrm{l}$ propidium-iodid. Mixtures were gently vortexed and incubated for 15 minutes at room temperature in the dark. After the incubation $400 \mu \mathrm{l}$ of the $1 \mathrm{x}$ binding-buffer were added to each tube and the samples were analyzed within 1 hour by flow cytometry (CFlow6, Accuri).

\section{Immunofluorescence and confocal laser-scanning microscopy}

For immunofluorescence analysis, cells were cultivated on glass cover-slides and fixed in 4\% paraformaldehyde/PBS for 10 minutes, permeabilized with $0.2 \%$ Triton X 100 for 20 minutes and blocked in $100 \%$ FBS for 1 hour. Monoclonal mouse antibodies were used for detection of $\alpha$-tubulin (DM 1A, T-9026, Sigma) and $\gamma \mathrm{H} 2 \mathrm{AX}$ (JBW301, Upstate) and Alexa Flour 555-conjugated anti-Mouse IgG was used (Invitrogen) as secondary antibody. Chromatin was stained by DAPI (Roth). Slides were covered with ProLong Gold antifade (Invitrogen). As a negative control all stainings were performed without primary antibody.

LSM (laser scanning microscopy) images were captured with a confocal microscope (LSM 700, Zeiss) using a Plan Apochromat 20x/0.8 M27 objective, ZEN 2009 software (Zeiss) and the following settings: image size $2048 \times 2048$ and 16 bit; pixel/dwell of $25.2 \mu$ s; pixel size $0.31 \mu \mathrm{m}$; laser power $2 \%$; master gain $600-1000$. After image capturing the original LSM files were converted into TIF files.

\section{Statistical analysis}

Statistical significance was determined using unpaired two tailed Student's t tests.

\section{Supplemental Information}

Supplement Information includes supplemental Movie S1 and Table S1. 


\section{ACKNOWLEDGEMENTS}

We thank Bruno Amati for kindly providing the pBabe-MYC-ER plasmid. This study was supported by grants to $\mathrm{HH}$ by the DFG/Deutsche Forschungsgemeinschaft, the Deutsche Krebshilfe, DKTK/German Consortium for Translational Cancer Research and the Rudolf-Bartling-Stiftung.

\section{Editorial note}

This paper has been accepted based in part on peerreview conducted by another journal and the authors' response and revisions as well as expedited peer-review in Oncotarget

\section{Disclosure of Potential Conflicts of Interest}

No potential conflicts of interest were disclosed.

\section{REFERENCES}

1. Atchley WR and Fitch WM. A natural classification of the basic helix-loop-helix class of transcription factors. Proc Natl Acad Sci U S A. 1997; 94(10):5172-5176.

2. Mermod N, Williams TJ and Tjian R. Enhancer binding factors AP-4 and AP- 1 act in concert to activate SV40 late transcription in vitro. Nature. 1988; 332(6164):557-561.

3. Imai $\mathrm{K}$ and Okamoto $\mathrm{T}$. Transcriptional repression of human immunodeficiency virus type 1 by AP-4. J Biol Chem. 2006; 281(18):12495-12505.

4. Kim MY, Jeong BC, Lee JH, Kee HJ, Kook H, Kim NS, Kim YH, Kim JK, Ahn KY and Kim KK. A repressor complex, AP4 transcription factor and geminin, negatively regulates expression of target genes in nonneuronal cells. Proc Natl Acad Sci U S A. 2006; 103(35):13074-13079.

5. Tsujimoto $K$, Ono $T$, Sato $M$, Nishida $T$, Oguma $T$ and Tadakuma T. Regulation of the expression of caspase- 9 by the transcription factor activator protein- 4 in glucocorticoidinduced apoptosis. J Biol Chem. 2005; 280(30):27638-27644.

6. Hu YF, Luscher B, Admon A, Mermod N and Tjian R. Transcription factor AP-4 contains multiple dimerization domains that regulate dimer specificity. Genes Dev. 1990; 4(10):1741-1752.

7. Jackstadt R, Roh S, Neumann J, Jung P, Hoffmann R, Horst D, Berens C, Bornkamm GW, Kirchner T, Menssen A and Hermeking H. AP4 is a mediator of epithelial-mesenchymal transition and metastasis in colorectal cancer. The Journal of experimental medicine. 2013; 210(7):1331-1350.

8. Jung P, Menssen A, Mayr D and Hermeking H. AP4 encodes a c-MYC-inducible repressor of $\mathrm{p} 21$. Proceedings of the National Academy of Sciences of the United States of America. 2008; 105(39):15046-15051.
9. Jung P and Hermeking H. The c-MYC-AP4-p21 cascade. Cell cycle. 2009; 8(7):982-989.

10. Jackstadt R, Jung P and Hermeking H. AP4 directly downregulates p16 and $\mathrm{p} 21$ to suppress senescence and mediate transformation. Cell death \& disease. 2013; 4:e775.

11. Hu BS, Zhao G, Yu HF, Chen K, Dong JH and Tan JW. High expression of AP-4 predicts poor prognosis for hepatocellular carcinoma after curative hepatectomy. Tumour Biol. 2012; 34(1):271-6.

12. Xinghua L, Bo Z, Yan G, Lei W, Changyao W, Qi L, Lin Y, Kaixiong T, Guobin $\mathrm{W}$ and Jianying $\mathrm{C}$. The overexpression of AP-4 as a prognostic indicator for gastric carcinoma. Med Oncol. 2012; 29(2):871-877.

13. Shi L, Jackstadt R, Siemens H, Li H, Kirchner $\mathrm{T}$ and Hermeking H. p53-induced miR-15a/16-1 and AP4 form a double-negative feedback loop to regulate epithelialmesenchymal transition and metastasis in colorectal cancer. Cancer Res. 2014; 74(2):532-542.

14. D'Annibale S, Kim J, Magliozzi R, Low TY, Mohammed S, Heck AJ and Guardavaccaro D. Proteasome-dependent Degradation of Transcription Factor Activating Enhancerbinding Protein 4 (TFAP4) Controls Mitotic Division. J Biol Chem. 2014; 289(11):7730-7737.

15. Dang CV, O'Donnell KA, Zeller KI, Nguyen T, Osthus RC and Li F. The c-Myc target gene network. Semin Cancer Biol. 2006; 16(4):253-264.

16. Adhikary $\mathrm{S}$ and Eilers M. Transcriptional regulation and transformation by Myc proteins. Nat Rev Mol Cell Biol. 2005; 6(8):635-645.

17. Alexandrow MG, Kawabata M, Aakre M and Moses HL. Overexpression of the c-Myc oncoprotein blocks the growth-inhibitory response but is required for the mitogenic effects of transforming growth factor beta 1. Proc Natl Acad Sci U S A. 1995; 92(8):3239-3243.

18. Meyer N, Kim SS and Penn LZ. The Oscar-worthy role of Myc in apoptosis. Semin Cancer Biol. 2006; 16(4): 275-287.

19. Berns K, Hijmans EM, Koh E, Daley GQ and Bernards R. A genetic screen to identify genes that rescue the slow growth phenotype of c-myc null fibroblasts. Oncogene. 2000; 19(29):3330-3334.

20. Philipp A, Schneider A, Vasrik I, Finke K, Xiong Y, Beach D, Alitalo K and Eilers M. Repression of cyclin D1: a novel function of MYC. Mol Cell Biol. 1994; 14(6):4032-4043.

21. Bouchard C, Thieke K, Maier A, Saffrich R, Hanley-Hyde J, Ansorge W, Reed S, Sicinski P, Bartek J and Eilers M. Direct induction of cyclin D2 by Myc contributes to cell cycle progression and sequestration of p27. EMBO J. 1999; 18(19):5321-5333.

22. Hermeking H, Rago C, Schuhmacher M, Li Q, Barrett JF, Obaya AJ, O'Connell BC, Mateyak MK, Tam W, Kohlhuber F, Dang CV, Sedivy JM, Eick D, Vogelstein B and Kinzler KW. Identification of CDK4 as a target of c-MYC. Proc Natl Acad Sci U S A. 2000; 97(5):2229-2234. 
23. Galaktionov K, Chen $\mathrm{X}$ and Beach D. Cdc25 cellcycle phosphatase as a target of c-myc. Nature. 1996; 382(6591):511-517.

24. Menssen A, Epanchintsev A, Lodygin D, Rezaei N, Jung P, Verdoodt B, Diebold J and Hermeking H. c-MYC Delays Prometaphase by Direct Transactivation of MAD2 and BubR1: Identification of Mechanisms Underlying c-MYCInduced DNA Damage and Chromosomal Instability. Cell Cycle. 2007; 6(3):339-352.

25. Felsher DW, Zetterberg A, Zhu J, Tlsty T and Bishop JM. Overexpression of MYC causes p53-dependent G2 arrest of normal fibroblasts. Proc Natl Acad Sci U S A. 2000; 97(19):10544-10548.

26. Brooks EE, Gray NS, Joly A, Kerwar SS, Lum R, Mackman RL, Norman TC, Rosete J, Rowe M, Schow SR, Schultz PG, Wang X, Wick MM and Shiffman D. CVT-313, a specific and potent inhibitor of CDK2 that prevents neointimal proliferation. J Biol Chem. 1997; 272(46):29207-29211.

27. Ewald B, Sampath D and Plunkett W. H2AX phosphorylation marks gemcitabine-induced stalled replication forks and their collapse upon S-phase checkpoint abrogation. Mol Cancer Ther. 2007; 6(4):1239-1248.

28. Berthet C, Aleem E, Coppola V, Tessarollo L and Kaldis P. Cdk2 knockout mice are viable. Curr Biol. 2003; 13(20):1775-1785.

29. Ortega S, Prieto I, Odajima J, Martin A, Dubus P, Sotillo R, Barbero JL, Malumbres $M$ and Barbacid M. Cyclindependent kinase 2 is essential for meiosis but not for mitotic cell division in mice. Nat Genet. 2003; 35(1):25-31.
30. Rane SG, Dubus P, Mettus RV, Galbreath EJ, Boden G, Reddy EP and Barbacid M. Loss of Cdk4 expression causes insulin-deficient diabetes and $\mathrm{Cdk} 4$ activation results in beta-islet cell hyperplasia. Nat Genet. 1999; 22(1):44-52.

31. Tsutsui T, Hesabi B, Moons DS, Pandolfi PP, Hansel KS, Koff A and Kiyokawa H. Targeted disruption of CDK4 delays cell cycle entry with enhanced p27(Kip1) activity. Mol Cell Biol. 1999; 19(10):7011-7019.

32. Normand G and King RW. Understanding cytokinesis failure. Adv Exp Med Biol. 2010; 676:27-55.

33. Adon AM, Zeng X, Harrison MK, Sannem S, Kiyokawa H, Kaldis $\mathrm{P}$ and Saavedra HI. Cdk2 and Cdk4 regulate the centrosome cycle and are critical mediators of centrosome amplification in p53-null cells. Mol Cell Biol. 2010; 30(3):694-710.

34. Doxsey S. Duplicating dangerously: linking centrosome duplication and aneuploidy. Mol Cell. 2002; 10(3):439-440.

35. Fukasawa K. Centrosome amplification, chromosome instability and cancer development. Cancer Lett. 2005; 230(1):6-19.

36. McDermott KM, Zhang J, Holst CR, Kozakiewicz BK, Singla V and Tlsty TD. p16(INK4a) prevents centrosome dysfunction and genomic instability in primary cells. PLoS biology. 2006; 4(3):e51.

37. Frank SR, Schroeder M, Fernandez P, Taubert S and Amati B. Binding of c-Myc to chromatin mediates mitogen-induced acetylation of histone H4 and gene activation. Genes Dev. 2001; 15(16):2069-2082. 